\title{
MadGolem: automated NLO predictions for Beyond-the-Standard Model searches
}

\author{
David López-Val*, Dorival Gonçalves-Netto, Tilman Plehn \\ Institut für Theoretische Physik, Universität Heidelberg \\ E-mails: lopez@thphys.uni-heidelberg.de \\ netto@thphys. uni-heidelberg.de \\ plehn@thphys.uni-heidelberg.de
}

\section{Kentarou Mawatari}

Theoretische Natuurkunde and IIHE/ELEM, Vrije Universiteit Brussel, Belgium

International Solvay Institutes, Brussels, Belgium

E-mail: kentarou.mawatarievub.ac.be

\section{loan Wigmore}

SUPA, School of Physics \& Astronomy, The University of Edinburgh, UK

E-mail: i.t.wigmoreded.ac.uk

\begin{abstract}
TeV-scale new physics is already at the spotlight of the current experimental LHC program. In this context, particle phenomenologists are urged to develop automated tools aiming at fast, flexible and accurate theoretical predictions for the major new physics discovery channels. In this contribution we provide a summarized description of the MADGOLEM package, a fully automated tool that enables to evaluate next-to-leading order (NLO) cross sections and distributions for generic heavy particle pair production beyond the Standard Model (BSM). We concisely portray the structure and inner architecture of the code, with our main emphasis on the strategies by which we handle the genuine building blocks of a NLO calculation - the evaluation of the one-loop amplitudes, the renormalization of the ultraviolet singularities, and the subtraction of infrared and on-shell divergences. We discuss the performance of the tool, its multiple functionalities and analysis options, and the attentive cross-checks of all these features. Finally, we present some sample applications to BSM heavy particle production at the LHC.
\end{abstract}

Prospects for Charged Higgs Discovery at Colliders - Charged 2012,

October 8-11, 2012

Uppsala University, Sweden

\footnotetext{
* Speaker.
} 


\section{MadGolem - a word of motivation}

The presence of colored heavy resonances constitutes a telltale prediction within many of the proposed extensions of the Standard Model (SM). Representative examples comprise e.g. the squarks and gluinos in supersymmetric (SUSY) theories; colored Kaluza-Klein excitations in extra dimensions; or colored composites from TeV-scale strongly-interacting sectors. With the LHC close to complete its $8 \mathrm{TeV}$ run, experimental searches have already set out on the quest for fingerprints of these hitherto hypothetical states. A most pressing demand at this stage is hence to improve the available theoretical predictions for the major doorways towards new physics discoveries. These should not only include the Next-to-Leading Order (NLO) QCD effects - which we know to have a remarkable impact, e.g. in the form of large radiative corrections and reduced theoretical uncertainties -, but also support a fast and flexible evaluation, so that these predictions can be readily exported to different models and parameter configurations.

In timely response to this imperative request, we present the package MADGOLEM [1, 2, 3, 4], by which we completely automate the calculation of NLO cross sections and distributions for $2 \rightarrow 2$ processes within generic beyond-the-Standard-Model (BSM) scenarios. The code is conceived as an independent, modular-structured add-on to MADGRAPH [5], and can thus benefit from its user interfaces, multiple operation possibilities and complementary analysis tools.

Following the final testing phase which is currently underway, we plan MADGOLEM to be publicly released and become an instrumental resource for model-building and phenomenology studies, and most specially for the LHC experimental community.

\section{MadGolem - code structure and functionalities}

The core structure and flowchart of the MADGoLEM code we sketch in Fig. 1. To begin with, we employ MADGRAPH [5] to generate all tree-level amplitudes, which we then cast into helicity amplitudes, according to HELAS [6]. Simultaneously we generate the matrix elements for the real emission NLO corrections. These include all channels with a three-particle final state, describing the initial/final state radiation of a light QCD parton. The NLO virtual corrections - stemming from the one-loop interchange of virtual colored particles - we first obtain with the aid of QGRAF [7], and we further manipulate analytically through a dedicated ensemble of FORM and MAPLE routines, which rely on color flow decomposition and spinor helicity techniques [8]. Tensor structures we handle by means of a modified Passarino-Veltman reduction scheme, as implemented in GoLEM [9]. These steps lead to a final result which we cast in terms of i) a set of partial amplitudes, viz. of coefficients tracking down the dependences on the masses, coupling constants and Mandelstam variables); and ii) a basis of $S U(3)_{C}$ group invariants, fundamental helicity structures, and one loop integrals. The latter we link to the ONELOOP library [10] for their numerical evaluation.

The resulting NLO amplitude entails, in addition, divergent contributions of various nature whose automated treament is perhaps the major hurdle to surmount in the design and implementation of the code. Ultraviolet (UV) singularities we renormalize over a suitable set of counterterms, whose Feynman diagrams and related amplitudes we again get from QGRAF. These expressions hinge on $\mathscr{O}\left(\alpha_{s}\right)$, model-dependent two-point functions, which we include within a separate library. Therewith we apply the $\overline{\mathrm{MS}}$ scheme for the renormalization of the strong coupling constant. We 


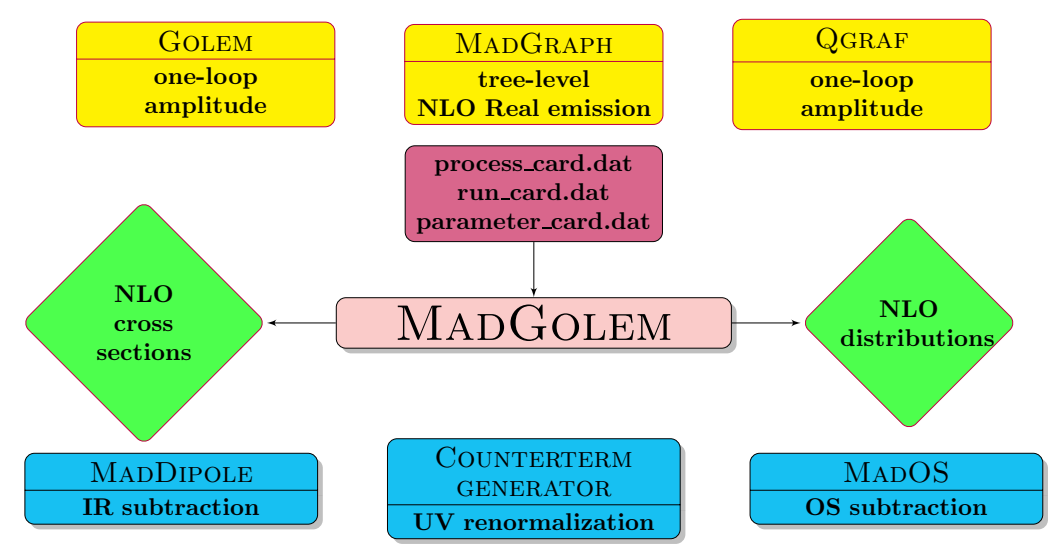

Figure 1: Schematic flowchart describing the MADGOLEM implementation.

consider its Renormalization Group running with five active flavors and explicitly decouple the heavy colored states [11]. The particle masses we anchor on-shell. Finite supersymmetry-restoring counterterms, if applicable, are automatically introduced at this point [12]. For the Infrared (IR) divergences we resort to the Catani-Seymour (CS) subtraction method [13]. We extend the MADDIPOLE package[14], in such a way that it now includes the novel massive CS dipoles needed to subtract the genuine IR structures emerging from non-standard massive colored fields (e.g. squarks, gluinos or sgluons, among others). Our implementation retains the dependence on the FKS-like $\alpha$ phase space cutoff [15], that separates the soft and collinear regions covered by the integrated dipoles (and hence adscribed to the virtual corrections) and those belonging to the $2 \rightarrow 3$ real emission process. Finally, we must remove potential divergences in the three-body phase space, occurring if the produced heavy resonances give rise to light-quark jets within the real emission process. These situations may lead to non-integrable phase space singularities and constitute a potential source of double counting, if not properly detached from the genuine NLO effects when the resonances are produced on shell. In order to subtract these divergences we stick to the PROSPINO scheme [16] and remove them locally over the entire phase space, preserving the gauge invariance as well as the spin correlations traded by the matrix elements. The corresponding subtraction terms have a Breit-Wigner shape and are generated in a completely automated fashion.

Based on alternative strategies to compute the NLO matrix elements and deal with the various divergent terms, a number of complementary approaches to automated NLO tools are presently under developement [17]. In this context, one very distinctive feature of MADGOLEM concerns the Feynman-diagrammatic description of the one-loop amplitudes, thanks to which the user has access to, and may retrieve, the results analytically all the way through the entire calculation. It also allows for an explicity selection and/or splitting of the different subsets of gauge-invariant one-loop topologies: self-energies, wave functions, boxes, and vertex corrections to the different interactions.

Moreover, MADGOLEM is endowed with a number of dedicated features which are particularly tailored to sweep the vast BSM scenery. Among them, we should highlight i) the use of fully flexible, unrestricted sets of inputs, with no default parameter space restrictions (unlike e.g. the 
mass degeneracy condition for the SUSY spectra that is assumed in PROSPINO); ii) the possibility to retrieve the results either as inclusive quantities or, instead, to disentangle the different initial and final-state contributions; iii) custom-built coding strategies, such as loop filtering, the grouping of topologically equivalent diagrams, and the intensive use of dynamically-linked libraries and multithread processing, all of them pursuing an optimal performance of the code - in particular concerning the size of the final executables and the overall running times; iv) the ability to cope with a broad variety of characteristic BSM structures, such as the presence of Majorana fermions or of non-renormalizable interactions descending from effective higher-dimensional operators.

The MADGOLEM code has been submitted to throughout cross-checks of manifold nature, involving e.g. the performance and numerical stability of the IR and OS subtraction procedures; the analytical and numerical cancellation of the UV and IR singularities; the finite parts of the renormalized one-loop amplitudes, which we have evaluated for a wide variety of representative processes and further contrasted to an independent calculation within FEYNARTS/FORMCALC/LOOPTOOLS [18]; and the distributions, which we have systematically compared to MADFKS [19]. Additionally, and whenever available, we have found satisfactory agreement between the MADGoLEM results and those derived either from PROSPINO and/or from independent predictions documented in the literature.

\section{MadGolem in operation}

With all this machinery set up to work, a systematic NLO description of BSM processes is now at reach and nails down to very few commands, by which the user can govern the different MADGOLEM functionalities. Aside from the evaluation of the total rates and $K$-factors, the design of MADGOLEM is particularly adequate for: i) portraying the NLO effects across the whole parameter space of a given new physics model, as no constraints nor relations among couplings and/or masses are assumed a priori; ii) describing the anatomy of the NLO quantum effects, through the separation of the distinct one-loop topologies and partonic subchannels; iii) quantifying the impact of the theoretical uncertainties, reflected in the sensitivity with respect to variations of the (unphysical) renormalization/factorization scales - whose value we can dial independently; iv) generating parton-level distributions for the main kinematic variables.

As a modular add-on to MADGRAPH, MADGOLEM profits from its standardized user interfaces, based on a set of input cards by which the process, model and collider setup may be fully set along. Furthermore, our package may be comfortably linked to the multiple complementary tools built upon the basic MAdGRAPH architecture - e.g. FeynRules, MADANALYSis, or MADWEIGHT [20].

Along these lines, a set of novel predictions for genuine new physics processes we have recently made available, including i) the associated production of squarks and EW gauginos in the MSSM [1]; ii) the production of scalar color-octet (sgluon) pairs [2]; and iii) the different squark and gluino pairwise channels [3]. All these qualify as pioneering examples of fully automated BSM calculations, and expand in many ways the possibilities at reach for the state-of-the-art (modeldependent, hard-coded) NLO packages. Some sample results we illustrate in Figure 2. 

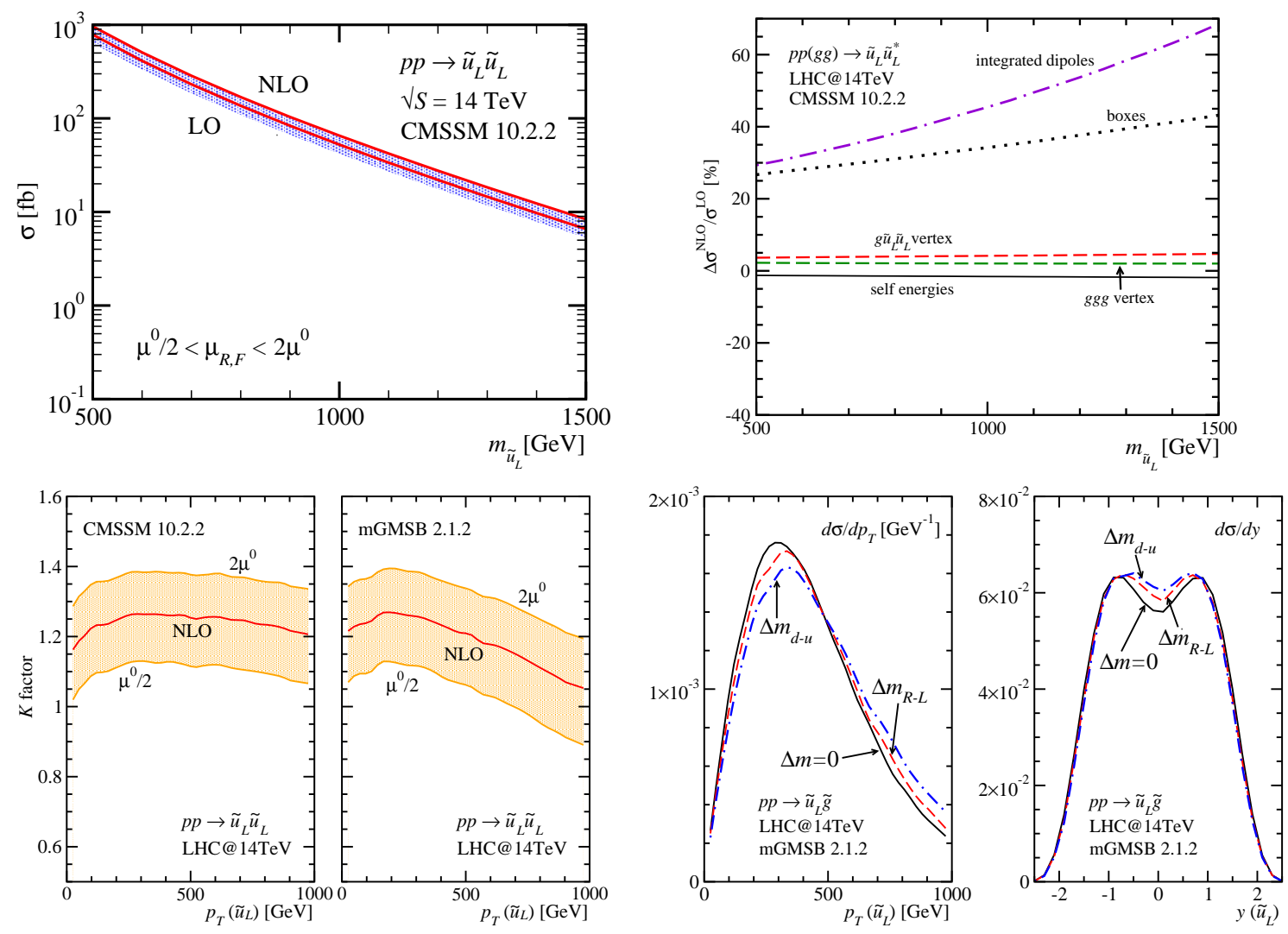

Figure 2: Sample of phenomenological analyzes carried out within MADGoLEM, taken from Ref. [3]. Top-left: total LO and NLO rates for squark-squark production $p p \rightarrow \tilde{u}_{L} \tilde{u}_{L}$ as a function of the squark mass

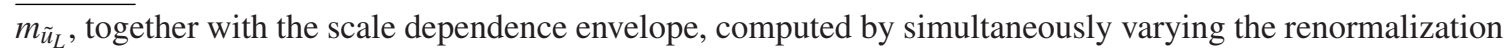
and factorization scales around the central value $\mu^{0} / 2<\mu_{R, F}<2 \mu^{0}$ (with $\mu^{0}=m_{\tilde{u}_{L}}$ ); top-right: relative size of the distinct NLO virtual corrections $\Delta \sigma^{\mathrm{NLO}} / \sigma^{\mathrm{LO}}$ from each of the topologies that contribute to the squark-antisquark production $p p(g g) \rightarrow \tilde{u}_{L} \tilde{u}_{L}^{*}$ at NLO. We single out the $g g$ partonic subchannel and display the results as a function of the squark mass $m_{\tilde{u}_{L}}$; bottom-left: $K$ factor as a function of $p_{T}\left(\tilde{u}_{L}\right)$ for squark-pair production $p p \rightarrow \tilde{u}_{L} \tilde{u}_{L}$, together with the scale dependence envelope, which we compute as explained above; bottom-right: normalized $p_{T}\left(\tilde{u}_{L}\right)$ and $y\left(\tilde{u}_{L}\right)$ distributions for squark-gluino production $p p \rightarrow \tilde{u}_{L} \tilde{g}$, where we compare the differences between i) degenerate squark masses $(\Delta m=0)$; ii) an overall left/right squark mass splitting $\left(\Delta m_{R-L}=200 \mathrm{GeV}\right)$; iii) likewise, but for down-up squarks $\left(\Delta m_{d-u}=200 \mathrm{GeV}\right)$. All plots we derive for the LHC running at $\sqrt{S}=14 \mathrm{TeV}$ and we entertain various MSSM benchmarks [21], as explicitly indicated in the figures.

From the compared study of all these outcomes we can read off a number of generic features which characterize the production of BSM heavy colored particle pairs at the LHC: i) The total rates are typically large (in the ballpark of $\sigma \sim 0.1-1 \mathrm{pb}$ for $\mathscr{O}(\mathrm{TeV})$ particles, and show a stark dependence on the final-state masses; ii) the corresponding NLO quantum effects are very significant, and lead to sizable $K$-factors $(\sim 1.5-2)$ - which, unlike the total rates, are barely sensitive to the details of the new physics mass spectrum; iii) the bulk NLO corrections arise from the one-loop exchange of virtual gluons and the initial/final state radiation of light QCD partons, whereas the new colored resonances - typically featuring mass-suppressed contributions - stamp a 
subdominant imprint; iv) the theoretical uncertainties are markedly reduced, which translates into a milder sensitivity under variations of the renormalization and factorization scales; v) the NLO distributions for a given process tend to be in excellent concordance with those derived independently from a multi-jet merging calculation, with the mere LO matrix elements being matched to a parton shower; vi) quite remarkably, we also find that the $K$-factors remain stable and relatively constant across the relevant phase space regions - an observation that supports the conventional practice of reweighting the Monte Carlo simulations with an overall $K$-factor from the full-fledged NLO yields.

Summing up, MADGOLEM enables a completely automated calculation of NLO cross sections and distributions, setting sights at the production of BSM particle pairs within generic new physics models. It represents, therefore, a major shortage between the BSM theory landscape and the NLO predictions for collider observables. The package has now reached a fully operative stage and will be publicly released in the course of the coming months.

Acknowledgements DLV wishes to thank the organizers of the Charged Higgs 2012 workshop for the opportunity to present our tool and for the lively atmosphere we all shared in Uppsala. Travel support is also gratefully acknowledged.

\section{References}

[1] T. Binoth, D. Gonçalves-Netto, D. López-Val, K. Mawatari, T. Plehn, and I. Wigmore, Phys. Rev. D 84, 075005 (2011).

[2] D. Gonçalves-Netto, D. López-Val, K. Mawatari, T. Plehn and I. Wigmore, Phys. Rev. D 85, 114024 (2012);

[3] D. Gonçalves-Netto, D. López-Val, K. Mawatari, T. Plehn and I. Wigmore, arXiv:1211.0286 [hep-ph].

[4] D. López-Val, D. Gonçalves-Netto, K. Mawatari, T. Plehn and I. Wigmore, arXiv:1209.2797 [hep-ph].

[5] J. Alwall et al., JHEP 0709, 028 (2007).

[6] H. Murayama, I. Watanabe and K. Hagiwara, KEK-91-11.

[7] P. Nogueira, J. Comput. Phys. 105, 279 (1993).

[8] Cf. e.g. Z. Xu, D. -H. Zhang and L. Chang, Nucl. Phys. B 291, 392 (1987); L. J. Dixon, "Calculating scattering amplitudes efficiently," [hep-ph/9601359], and references therein.

[9] G. Cullen, N. Greiner, A. Guffanti, J. -P. Guillet, G. Heinrich, S. Karg, N. Kauer and T. Kleinschmidt et al., Nucl. Phys. Proc. Suppl. 205-206, 67 (2010); T. Binoth, J. P. Guillet, G. Heinrich, E. Pilon, and T. Reiter, Comput. Phys. Commun. 180, 2317 (2009); N. Greiner, A. Guffanti, T. Reiter and J. Reuter, Phys. Rev. Lett. 107, 102002 (2011); G. Cullen, J. P. .Guillet, G. Heinrich, T. Kleinschmidt, E. Pilon, T. Reiter and M. Rodgers, Comput. Phys. Commun. 182, 2276 (2011).

[10] A. van Hameren, Comput. Phys. Commun. 182, 2427 (2011).

[11] J. C. Collins, F. Wilczek, and A. Zee, Phys. Rev. D 18, 242 (1978); S. Berge, W. Hollik, W. M. Mösle, and D. Wackeroth, Phys. Rev. D 76, 034016 (2007).

[12] S. P. Martin and M. T. Vaughn, Phys. Lett. B 318, 331 (1993).

[13] S. Catani and M. H. Seymour, Phys. Lett. B 378, 287 (1996); S. Catani and M. H. Seymour, Nucl. Phys. B 485, 291 (1997) [Erratum-ibid. B 510, 503 (1998)]; S. Catani, S. Dittmaier, M. H. Seymour and Z. Trocsanyi, Nucl. Phys. B 627, 189 (2002). 
[14] R. Frederix, T. Gehrmann, and N. Greiner, JHEP 0809, 122 (2008); and JHEP 1006, 086 (2010).

[15] S. Frixione, Z. Kunszt, and A. Signer, Nucl. Phys. B 467, 399 (1996); Z. Nagy and Z. Trocsanyi, Phys. Rev. D 59, 014020 (1999) [Erratum-ibid. D 62, 099902 (2000)].

[16] See e.g. W. Beenakker, R. Höpker, M. Spira, and P. M. Zerwas, Phys. Rev. Lett. 74, 2905 (1995); W. Beenakker, R. Höpker, M. Spira and P. M. Zerwas, Nucl. Phys. B 492, 51 (1997); W. Beenakker, M. Krämer, T. Plehn, M. Spira and P. M. Zerwas, Nucl. Phys. B 515, 3 (1998).

[17] G. Cullen, N. Greiner, G. Heinrich, G. Luisoni, P. Mastrolia, G. Ossola, T. Reiter and F. Tramontano, Eur. Phys. J. C 72, 1889 (2012); V. Hirschi, R. Frederix, S. Frixione, M. V. Garzelli, F. Maltoni and R. Pittau, JHEP 1105, 044 (2011); G. Bevilacqua, M. Czakon, M. V. Garzelli, A. van Hameren, Y. Malamos, C. G. Papadopoulos, R. Pittau and M. Worek, Nucl. Phys. Proc. Suppl. 205-206, 211 (2010); C. F. Berger, Z. Bern, L. J. Dixon, F. Febres Cordero, D. Forde, H. Ita, D. A. Kosower and D. Maitre, Phys. Rev. D 78, 036003 (2008); T. Gleisberg, S. .Hoeche, F. Krauss, M. Schonherr, S. Schumann, F. Siegert and J. Winter, JHEP 0902, 007 (2009).

[18] T. Hahn, Comput. Phys. Commun. 140, 418 (2001); T. Hahn and M. Pérez-Victoria, Comput. Phys. Commun. 118, 153 (1999); T. Hahn and C. Schappacher, Comput. Phys. Commun. 143, 54 (2002); T. Hahn and M. Rauch, Nucl. Phys. Proc. Suppl. 157, 236 (2006).

[19] R. Frederix, S. Frixione, F. Maltoni and T. Stelzer, JHEP 0910, 003 (2009).

[20] P. Artoisenet, V. Lemaitre, F. Maltoni and O. Mattelaer, JHEP 1012, 068 (2010); C. Degrande, C. Duhr, B. Fuks, D. Grellscheid, O. Mattelaer and T. Reiter, Comput. Phys. Commun. 183, 1201 (2012); E. Conte, B. Fuks and G. Serret, arXiv:1206.1599 [hep-ph].

[21] S. S. AbdusSalam, B. C. Allanach, H. K. Dreiner, J. Ellis, U. Ellwanger, J. Gunion, S. Heinemeyer and M. Krämer et al., Eur. Phys. J. C 71, 1835 (2011) 